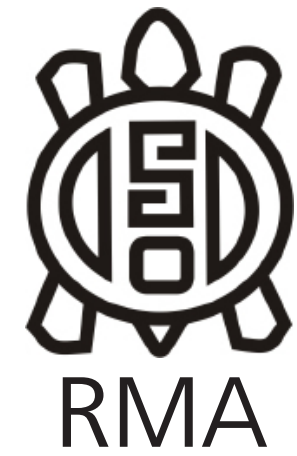

Arqueología

\title{
Relevamiento y análisis de materias primas arcillosas y su vinculación con la producción cerámica prehispánica del Valle de Tafí (Tucumán, Argentina)
}

Survey and analysis of clay raw materials and their link with the preHispanic ceramic production of the Tafi Valley (Tucumán, Argentina)

María Cecilia Páez* y Bárbara Manasse**

* CONICET, Museo de La Plata, Facultad de Ciencias Naturales y Museo, Universidad Nacional de La Plata, Argentina. E-mail: ceciliapaez@gmail.com

** Facultad de Ciencias Naturales e Instituto Miguel Lillo, Universidad Nacional de Tucumán; Escuela de Arqueología, Universidad Nacional de Catamarca.

E-mail: bamanasse@gmail.com

\begin{abstract}
Resumen
El Valle de Tafí presenta evidencias de ocupación prehispánica durante al menos 7500 años AP. Se trata de materialidades ancestrales que respaldan la profundidad temporal y la importancia socio-cultural y política de esta región. Hacia mediados del segundo milenio de la Era Cristiana las poblaciones locales entraron en la órbita de las políticas expansivas del Estado Inca. Aún sabemos poco sobre su incidencia en la configuración del paisaje, así como en la posible reorganización de al menos algunos sectores de la sociedad. Nos interesa evaluar, en este caso, la incidencia en la producción alfarera que, por lo pronto, se expresa en variedad tecnológica y decorativa. Con ese objetivo nos propusimos 1) identificar y relevar las fuentes arcillosas en el ámbito del Valle, 2) caracterizar estas arcillas, en vistas a determinar su aptitud para la manufactura alfarera, 3) correlacionar a nivel químico arcillas y fragmentos asignados al segundo milenio y 4) analizar las implicancias sociales y políticas de una producción alfarera local y/o no local. Los resultados obtenidos significan un avance en el conocimiento de la incidencia de expansión imperial en estos territorios.
\end{abstract}

Palabras clave: Relevamiento de arcillas; Análisis de Activación Neutrónica; Producción local de cerámica; Segundo Milenio prehispánico; Tafí del Valle.

\begin{abstract}
The Tafi Valley presents evidence of pre-Hispanic occupation for at least 7500 years BP. These are ancestral materialities that support the temporal depth and the socio-cultural and political importance of this region. Towards the middle of the second millennium of the Christian Era, local populations entered the orbit of the expansive policies of the Inca State. We still know little about its impact on the configuration of the landscape, as well as on the possible reorganization of at least some sectors of society. We are interested in evaluating, in this case, the incidence on pottery production, which, for the time being, is expressed in a technological and decorative variety. With this objective we set out 1) to identify and survey the clay sources in the Valley, 2) characterize these clays, in order to determine their suitability for pottery manufacturing, 3) correlate at a chemical level clays and fragments assigned to the second millennium and 4) analyze the social and political implications of local and / or non-local pottery production. The results obtained signify an advance in the knowledge of the incidence of imperial expansion in these territories.
\end{abstract}

Keywords: Clay survey; Neutron Activation Analysis; Local ceramic production; Pre-Hispanic Second Millennium; Tafí Valley.

\section{Introducción}

La ocupación de sociedades agropastoriles previa a la invasión española en el Valle de Tafí se refleja en una abundante cantidad de material alfarero que aflora en áreas de vivienda, espacios destinados a cultivos y pastoreo y/o de sentido ceremonial. Por más de dos mil años se fueron acumulando evidencias materiales de estos pueblos que habitaban la región de valles, quebradas y cerros hasta el monte del faldeo oriental de las Cumbres 
Calchaquíes. Se trata de un indicador importante para abordar la historia de los pueblos de estas regiones, sorteando - de acuerdo con la perspectiva teórica que guía nuestras investigaciones - interpretaciones esencialistas que relacionan estilos alfareros con etnicidad (Manasse 2014a, 2019, Páez y Giovannetti 2008, 2009). De hecho, los estudios que referiremos a continuación parten del supuesto de una significativa continuidad poblacional que poco permite "aislar" o "circunscribir" entidades culturales del tipo que clásicamente identifican lugares y tiempos prehispánicos.

Las investigaciones que venimos desarrollando se concentran en los mil años más recientes del pasado de Tafí, que remiten, sin lugar a duda, al de los Valles Calchaquíes en general, así como al del área occidental de las yungas (Manasse 2012, 2019). Se trata de tiempos sólo recientemente "incorporados" a la "historia" de la región. Aún se discute el rol de estos espacios orientales en un período que se destaca por el desarrollo de sociedades de organización política y territorial compleja (Manasse 2017). En este contexto, es aún menos lo que sabemos sobre cómo fue integrado este valle de altura al dominio imperial cuzqueño. La falta de arquitectura típica del incario hizo presuponer que esta región no había sido incluida en esa esfera (Cf. Raffino 1991) 1. Como lo señalara una de nosotras, es con nuestras investigaciones en el noreste del Valle de Tafí, en el pie de monte meridional de las Cumbres Calchaquíes, que pudimos conocer algo más sobre la dinámica de estas sociedades durante el siglo XV d. C (Manasse 2014b).

Algunos aspectos de interés, relacionados a la integración de estos territorios a la órbita imperial se vinculan con los modos en los que la población local fue "articulando" roles (en lo social, económico y político) y desarrollando resistencias; con las funciones y los lugares que pudieron haber sido impuestos para cumplir con los mandatos de las autoridades. Ello implica las relaciones entre la población local y los representantes del Cuzco, pero también, dentro de la propia sociedad nativa del valle. De hecho, las características de esta última, para el Tardío regional, han sido discutidas en función de presupuestos que interpretan al Valle de Tafí conformando lo que sería el "borde oriental" de la región valliserrana, habiendo funcionado como un espacio periférico, aunque complementario: desde lo ecológico, en los primeros siglos del segundo milenio EC, y particularmente desde lo geopolítico, en tiempos del incario (cf. Núñez Regueiro 1972; Sempé 1999). Lo estamos discutiendo hace varios años atrás (Manasse 2012).

En la actualidad no quedan dudas de que Tafí integró territorio diaguita (Manasse 2019 y textos allí citados). Con asentamientos en distintas partes del valle, además

\footnotetext{
${ }^{1}$ Recién en una edición mucho más reciente, Rodolfo Raffino (2007) integra al Valle de Tafí en la esfera imperial inca, tomando como referencia uno de los sitios que vamos a presentar en este artículo.
}

de la zona serrana, los fechados indican coetaneidad con los procesos históricos de los valles occidentales (Yocavil, Calchaquí). Nos interesa ahondar en la investigación de las estrategias desarrolladas en relación al dominio de estos territorios por parte del imperio cuzqueño y la resistencia y las negociaciones que pudieron haber desplegado los habitantes nativos del valle. Para avanzar en un problema que, sin duda, requiere un estudio amplio y diverso, nos enfocamos en este caso en el análisis de ciertas características de la producción cerámica de las sociedades nativas que habitaron el Valle en el último milenio EC. A tal fin, abordaremos el análisis de alfarería proveniente de contextos previos a la integración del valle al dominio inca como otras, de estos momentos. Atendiendo a experiencias en estos mismos sentidos como aquellos más centrados en aspectos estilísticos (cf. Calderari y Williams 1991; Marchegiani et al 2009) o, también, tecnológicos (cf. Cremonte 2006; Vera et al., 2019) - venimos efectuando estudios petrográficos, que están dando cuenta de la utilización de una variedad de técnicas para la preparación de las pastas. Observamos la mezcla de arcilla con arenas locales -como es el caso de la cerámica de estilo Tafí y la alfarería tosca asociada al segundo milenio EC -, o el empleo de cerámica molida y componentes volcánicos (Páez, 2010 a y b; 2013; Páez y Arnosio, 2009). Estas técnicas no son exclusivas del Valle, por el contrario, guardan correspondencia con tecnologías implementadas en otras regiones del Noroeste argentino (cf. Cremonte y Pereyra Domingorena, 2013; Iucci 2013; Páez et al., 2013; Palamarczuk et al., 2014; Pérez y Tchilinguirian, 2016; Prieto Olavarría y Páez, 2015; Puente, 2011; Zagorodny et al. 2010).

La alfarería recuperada en contextos arqueológicos del segundo milenio EC en el Valle de Tafí se puede identificar con los estilos decorativos Santa María (en sus variantes bicolor, tricolor y negro sobre rojo), Belén, Famabalasto negro sobre rojo y negro grabado, Yocavil Polícromo e Inca, en asociaciones semejantes (en cuanto a diversidad de expresiones alfareras) al de varias localidades de los Valles Calchaquíes. Tomando en cuenta el valor cultural que la alfarería ha tenido en los primeros siglos del segundo milenio prehispánico, así como su peso político en tiempos de la expansión imperial, nos abocamos inicialmente a la investigación de procesos implicados en su producción que pudieran darnos una pauta acerca de las modalidades y sujetos implicados.

Con el fin de cotejar la posibilidad de una producción local (con materias primas propias de este Valle) de piezas alfareras de aquellos distintos estilos se procedió, en primera instancia, a la identificación de posibles fuentes de materia prima que, por sus condiciones y características, sean aptas para el trabajo alfarero (vgr. Williams y Cremonte 1994). Ello comprendió particularmente el reconocimiento de bancos de arcillas en el ámbito del Valle de Tafí y su evaluación en términos de potencialidad para la manufactura cerámica. En segundo lugar, se procedió a realizar la correlación química entre arcillas y fragmentos cerámicos hallados 
en contextos prehispánicos más recientes, previos y contemporáneos a la incorporación a la esfera imperial.

\section{El paisaje del Valle de Tafí}

El valle de Tafí es una cuenca tectónica intermontana ubicada entre los 1850 y 2500 msnm, en el Oeste de la provincia de Tucumán (Figura 1), enclavada entre las Cumbres Calchaquíes al Norte, y las sierras de Aconquija, por el Sur y Oeste. Las cumbres de Mala Mala lo delimitan, siendo el nexo natural con la selvática llanura oriental, (Manasse, 2017). Hacia el Oeste, el cerro Muñoz - que forma parte del Sistema del Aconquija - presenta una serie de pasos hacia la región del Yocavil, siendo, además, las abras del Infiernillo o el de las Ánimas los accesos más claros desde el punto de vista geográfico. El uso de los mismos está atestiguado por los pobladores actuales que, aún hoy, recorren estos cerros, las altiplanicies de la cumbre y las quebradas, que finalmente permiten llegar a localidades como Ampajango, Entre Ríos, Los Colorados y Andalhuala, por ejemplo. Corresponde a áreas de tránsito que en el pasado eran aún más frecuentes y enlazaban las poblaciones de ambos valles. Su localización es, así, estratégica y sin lugar a dudas fue aprovechada antes y durante la expansión imperial cuzqueña.

Las ocupaciones prehispánicas más recientes se observan en una serie de núcleos poblacionales emplazados tanto en fondo de valle como en el pie de monte y los faldeos serranos. Estos se articulan con espacios productivos agropastoriles y otros, de fuerte sentido espiritual, político y estratégico (Manasse, 2014). Se los ubica en todo el valle;

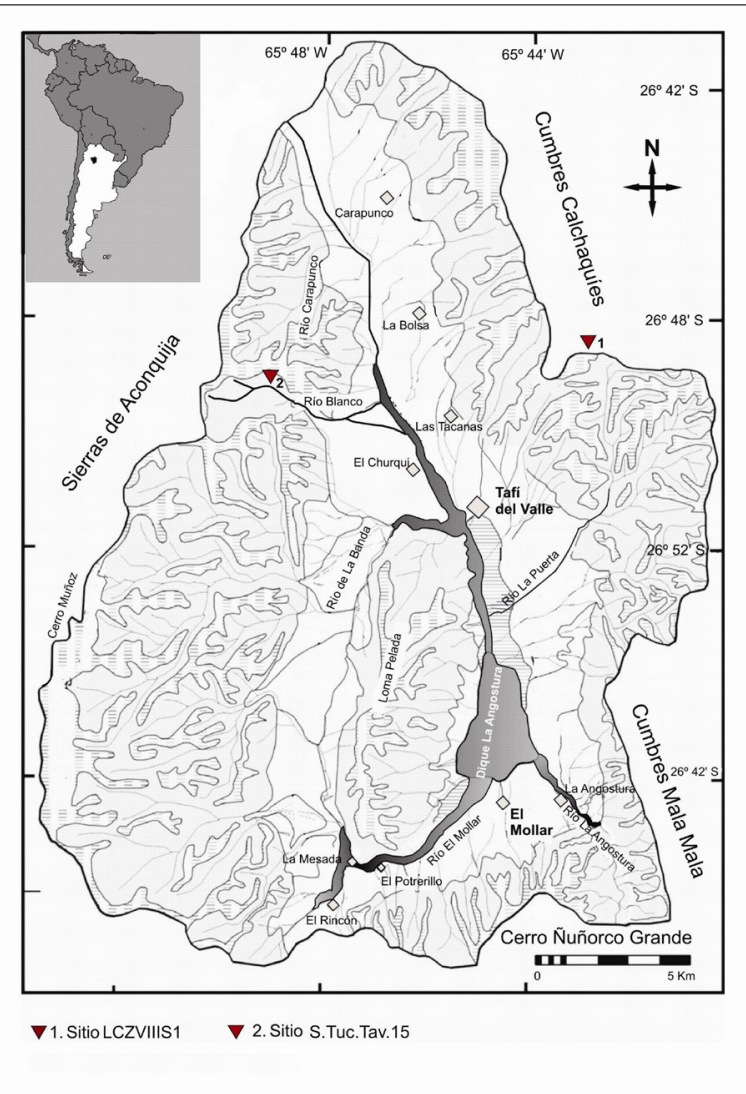

los hemos registrado también estrechamente vinculados a asentamientos de otros tiempos. En esta primera mitad del segundo milenio de la Era Cristiana se continuó habitando los mismos lugares poblados desde siglos atrás, sin generar su destrucción sino logrando su integración, al menos desde un punto de vista arquitectónico y del paisaje. Estos se suman, se funden. Son paisajes que denotan la búsqueda de una territorialización; tanto la arquitectura como otras materialidades dan cuenta de lo que podemos interpretar como construcción de continuidades con tiempos previos (Manasse, 2017). Algunas evidencias en áreas, como La Mesada de Los Teros, al Norte del Valle de Tafí, o la zona baja del cono del río La Puerta ( $B^{\circ}$ Malvinas), nos sugieren que este tipo de prácticas también continúan en tiempos en que el valle fuera integrado al espacio y a las dinámicas sociopolíticas del incario.

En el marco de un ambiente geológico de Sierras Pampeanas, la composición mineralógica del Valle incluye rocas metamórficas, plutónicas, de mezcla e hipabisales (Ruiz Huidobro, 1972), que se relacionan con la Formación Puncoviscana (Turner, 1960, 1972). Los depósitos mesozoicos y terciarios están compuestos de rocas sedimentarias como areniscas y material de origen volcánico. También se describen rocas piroclásticas, sobre todo de edad terciaria, y sedimentitas loesicas cuaternarias con intercalaciones de bancos de tobas (Fernández 1995). Los depósitos cuaternarios se caracterizan por sedimentos aluviales que constituyen secuencias de loes-paleosuelo de la Formación Tafí del Valle, alternando con conglomerados, fanglomerados y depósitos aluviales (Collantes et al., 1993).

Los depósitos arcillosos descriptos para el valle por Ruiz Huidobro (1972) incluyen tanto los residuales - es decir, formados a partir de la meteorización in situ del subsuelo rocoso - como secundarios, producto del transporte de agentes fluviales y eólicos. Ambos están presentes tanto en el fondo de la cuenca como hasta la parte media de las laderas que la circundan, en el Cerro Ñuñorco y en el Zanjón del Potrerillo. Las que están en el fondo de la cuenca y en las laderas tienen una coloración amarillo rojiza, al igual que las del cerro Ñuñorco. En el Zanjón del Potrerillo y en el río Tafí, frente a Carapunco, son más arenosas y están estratificadas, de acuerdo a las diferentes tonalidades visibles, con colores castaños y nódulos ocráceos. Esta información es de gran valor para confirmar la presencia de depósitos arcillosos en Tafí y se constituyó en un importante punto de partida para el relevamiento exhaustivo que requiere el estudio de la

Figura 1. Mapa de ubicación del valle de Tafí con los dos sitios arqueológicos de los cuales procede el registro cerámico analizado, S.Tuc.Tav. 15 y LCZVIIIS1.

Figure 1. Location map of the Tafi valley with the two archaeological sites from which the analyzed ceramic record comes, S.Tuc.Tav.15 and LCZVIIIS1. 
producción cerámica prehispánica.

Avanzaremos aquí en los estudios de alfarería proveniente de dos contextos diferentes pertenecientes a ocupaciones humanas de la primera mitad del segundo milenio d.C. (Figura 1). El sitio S.Tuc.Tav.15, también conocido como "El Linde", es un cementerio de tiempos preincaicos, en el que se registraron unos 40 entierros en urnas con clásica decoración santamariana. Se localiza en el noroeste del valle de Tafí, a los $26^{\circ} 51^{\prime} 19,3^{\prime \prime}$ lat.S y $65^{\circ} 45^{\prime} 00,0^{\prime \prime}$ long. W (Leiva Benegas, 2003), en una hondonada de la ladera de una profunda quebrada. El material cerámico recuperado se encontraba altamente fragmentado, dado que el sitio fue intervenido por saqueadores.

El segundo contexto corresponde al sitio LCZVIIIS1, denominado "Pucará de las Lomas Verdes" en la literatura arqueológica (Santillán de Andrés, 1951; Tarragó y Núñez Regueiro, 1972; Manasse, 2003). Se trata de un asentamiento circunscripto espacialmente en un morro localizado a unos 2500 msnm, al NNE del valle (a los $26^{\circ} 49^{\prime} 57,1^{\prime \prime}$ lat.S y 6541'30,7" long.W) .Se trata de un espacio delimitado natural y artificialmente con una excelente vista de buena parte del valle (Patané Aráoz, 2008). Nuestras investigaciones arqueológicas sugieren que puede haber sido construido en tiempos previos a la expansión imperial inca, pero fue ocupado durante esta última, cuando se habría desempeñado en vinculación con los sectores de poder local, a juzgar por las características del registro material. Los estudios efectuados dan cuenta de la realización de actividades de tipo congregativo y ritual (Manasse, 2014, Páez 2010). Los fragmentos alfareros recuperados en excavaciones y recolecciones de superficie corresponden a los estilos Santa María en diferentes variedades, Famabalasto negro sobre rojo y Famabalasto negro grabado, Yokavil Polícromo e Inca provincial (Calderari y Williams, 1991), además de fragmentos alisados y/o peinados/cepillados, sin decoración pintada.

Contamos así, con fragmentos de dos contextos cronológicamente diferentes (previo a la integración a la esfera imperial y coetánea a la misma) provenientes de localizaciones que, aunque distantes entre sí, habrían participado de la misma dinámica social y cultural.

\section{Metodología}

El abordaje de los objetivos requirió el trabajo sobre dos líneas de evidencia. Por un lado, sobre potenciales fuentes de aprovisionamiento de materias primas para la manufactura alfarera y, por el otro, sobre fragmentos cerámicos seleccionados de los contextos previamente descriptos.

La identificación de potenciales fuentes de arcillas se realizó apelando a datos geológicos y, fundamentalmente, a información brindada por pobladores del valle, algunos de les cuales elaboraban piezas alfareras con arcillas locales. A partir de ello, se realizaron una serie de relevamientos, georeferenciando y caracterizando los depósitos registrados desde su accesibilidad, visibilidad, geomorfología, fitogeografía, uso actual del sector y demás información que fuera de interés. Se utilizaron variables de registro geológico específicas, enfocadas al perfil edáfico del sector, los rasgos del afloramiento y las características estructurales y texturales de los estratos arcillosos (cf. Cuenya et al., 2007).

De esta manera, el trabajo sobre las fuentes de arcilla se realizó siguiendo las siguientes etapas: a) establecimiento de criterios y selección de las unidades geológicas favorables para hacer la toma de muestras, b) reconocimiento en campo de las unidades geológicas con el fin de determinar los diferentes estratos o niveles arcillosos, su continuidad lateral, potencia, grado de cementación y consistencia, además de la información arqueológica pertinente, $y, c)$ toma de muestras, abriendo canales de $10 \mathrm{~cm} \times 10 \mathrm{~cm} \times 10 \mathrm{~cm}$, perpendiculares a los estratos. La recolección fue convenientemente identificada en bolsas individuales con etiqueta, y registrada en planillas con un soporte fotográfico y d) análisis granulométrico y mineralógico de cada depósito.

Este último paso se efectuó en colaboración con la Dra. Patricia Cuenya y la Geól. Ramona Ovejero². Las muestras recolectadas fueron clasificadas de acuerdo a sus propiedades texturales siguiendo las pautas que se utilizan para el análisis de suelos. Se establecieron sus propiedades físicas (análisis granulométricos) y geomecánicas (límite líquido, límite plástico, índice plástico e índice de contracción lineal). El límite líquido (LL) es el pasaje del estado líquido al plástico, y se mide por la cantidad de agua que contiene el material en el momento en que se pierde la fluidez de un líquido denso. El limite plástico (LP) es el punto en el que se pasa del estado plástico al semisólido, y se mide por la cantidad de agua que contiene un material en el momento en que se pierde la plasticidad y deja de ser moldeable. La diferencia entre el Limite Liquido (LL) y el Limite Plástico (LP) define el Índice de Plasticidad (IP) (De Pablo, 1964). Por otra parte, el Coeficiente de Extensibilidad Lineal (COLE) permite evaluar la capacidad de ciertos suelos de expandirse cuando aumenta el contenido de humedad y agrietarse cuando este disminuye, y depende del contenido de arcillas expandibles (Brasher et al., 1966). Se clasifica como bajo, cuando el COLE es inferior a 3; moderado cuando va de 3 a 6; alto de 6 a 9 y muy alto cuando supera el valor de 9 (Thomas et al. 2000). Aquellas muestras con mayor contenido arcilloso manifestarían valores significativos en relación con este coeficiente. Los materiales aptos para la manufactura cerámica necesariamente deben comportarse de manera plástica en presencia de agua (índice de plasticidad, IP) y no experimentar variaciones

\footnotetext{
${ }^{2}$ Ambas, de la Facultad de Ciencias Naturales e Instituto Miguel Lillo, Universidad Nacional de Tucumán.
} 
sustanciales en su volumen durante el proceso de secado (límite de contracción lineal, COLE). Valores de IP hasta 20, porcentajes inferiores a 7 de COLE y caolinita como argilomineral dominante indican un material ideal para este fin.

Pero, más allá de estos factores, que tienen que ver con la composición y características sedimentarias, también hay otros aspectos importantes a tener en cuenta como posibles modificadores de la plasticidad de la arcilla. Tal es el caso, como ya lo indicamos en otra oportunidad ${ }^{3}$, del contenido orgánico que usualmente actúa como coloide, aumentando los niveles de plasticidad.

Finalmente, determinamos aspectos generales de la mineralogía (filosilicatos y no filosilicatos), mediante análisis de Difracción de Rayos X, que permite determinar estructuras cristalinas de composición química compleja y de baja simetría (Kraus et al., 1965). Los argilominerales se identificaron con un difractómetro Phillips PW 3719 con ánodo de Co del Instituto de Estratigrafía y Geología Sedimentaria Global (IESGLO), Facultad de Ciencias Naturales e Instituto Miguel Lillo, Universidad Nacional de Tucumán.

Con el fin de realizar una comparación más controlada entre muestras arcillosas y los fragmentos cerámicos prehispánicos, se elaboraron briquetas experimentales con una medida estándar de $6 \times 6 \times 1 \mathrm{~cm}$. con las muestras más aptas para el trabajo alfarero ${ }^{4}$. Ello también permitió evitar los inconvenientes de comparar químicamente arcillas cocidas con arcillas crudas. Tanto la elaboración de las briquetas como su cocción se realizaron bajo condiciones controladas. No se adicionó material antiplástico, pero se eliminaron las impurezas y clastos de tamaño medio y grande. Se agregó agua al sedimento para su hidratación, se colocó la preparación en una placa de yeso para que eliminara el agua sobrante y se procedió al amasado y elaboración de las briquetas. La temperatura de cocción se mantuvo en $850^{\circ} \mathrm{C}$ en horno eléctrico, de acuerdo a las estimaciones sobre los gradientes de calor a los que se habría sometido a las piezas cerámicas prehispánicas, atendiendo a los resultados de nuestros estudios y los análisis de Difracción de Rayos X (Páez 2010). La cocción se realizó en una atmósfera oxidante, tal como se observó desde el estudio de las pastas de los fragmentos de los contextos prehispánicos analizados.

El análisis químico de los fragmentos cerámicos de esos contextos prehispánicos se realizó sobre una muestra de cincuenta fragmentos $(n=50)$. Su selección apuntó a obtener una adecuada representatividad estilística, morfológica y tecnológica de cada sitio (Tabla 1).

\footnotetext{
${ }^{3}$ Cf. Cuenya et al., 2007.

${ }^{4}$ La selección priorizó estas muestras dado el número de estudios que se podían realizar en ese momento, sin que por ello consideremos, según lo explicamos más adelante en el texto, que las otras (salvo un caso) no hayan sido de utilidad para la manufactura e piezas cerámicas.
}

Briquetas y fragmentos cerámicos fueron sometidos a análisis de Activación Neutrónica (AAN) ${ }^{5}$, los que fueron realizados en el Laboratorio de Técnicas Analíticas Nucleares del Centro Atómico Ezeiza (Buenos Aires, Argentina). Las irradiaciones se realizaron en el reactor RA-3 (flujo térmico $3.10^{13} \mathrm{~cm}^{-2} . \mathrm{s}^{-1} ; 4,5 \mathrm{Mw}$ ) y se determinaron 20 elementos químicos que incluyen: As, $\mathrm{Ba}, \mathrm{Ce}, \mathrm{Co}, \mathrm{Cr}, \mathrm{Cs}, \mathrm{Eu}, \mathrm{Fe}, \mathrm{Gd}, \mathrm{Hf}$, La, Lu, Rb, Sb, Sc, Sm, $\mathrm{Tb}, \mathrm{Th}, \mathrm{U}$ y Yb. Los análisis estuvieron a cargo de la Dra. Rita Pla (Páez y Plá 2015).

\section{Fuentes de aprovisionamiento y análisis de las arcillas}

Hemos identificado ocho depósitos arcillosos $(n=8)$ en el Valle de Tafí que presentaban potencialidad de aprovechamiento. Los mismos se ubicaron en barrancas y cortes naturales, así como también en sectores abiertos en los márgenes de cursos de agua. Se obtuvo un total de once muestras $(n=11)$ para su análisis. La tabla 2 resume las características de las áreas muestreadas, su vinculación con las actividades actuales y el tipo de evidencia arqueológica identificada en las proximidades.

Sector 1 (S1): El área recibe el nombre de Zanjón del Chivo y se localiza en la localidad de La Angostura; se trata de un cono glacís atravesado por el río Los Sosa, actualmente sujeto a un intenso proceso de carcavamiento. Sobre el perfil de la barranca donde se extrajo la muestra (M1) hay evidencias de socavamiento antrópico, probablemente vinculado a la extracción de muestras para uso geológico. El sedimento extraído es de color 10YR 7/4, de textura limo arenosa, con rodados de cuarcita de tamaño mayor a 0,01 m. y forma subangulosa. Dentro del mismo estrato se observaron estructuras químicas, probablemente carbonáticas.

Sector 2 (S2): Está ubicado, al igual que S1, en La Angostura, en el faldeo occidental de las Cumbres Mala Mala, en un sector denominado Zanja de la Loma Bola. Conforma un estrato muy visible, con una potencia de aprox. $1 \mathrm{~m}$ de espesor en el perfil de una barranca. Este afloramiento vuelve a aparecer a algunos metros de distancia (S3). Forma una cubierta de meteorización del granito, que se observa como manchones rojos en cárcavas. Las estructuras de expansión y contracción visibles, presentes en la superficie, dan una idea de su

\footnotetext{
${ }^{5}$ La técnica consiste en irradiar la muestra y un blanco de composición conocida con un flujo de neutrones térmicos (neutrones lentos), generando reacciones nucleares. La absorción por parte del núcleo de un neutrón da lugar a un radioisótopo que en un momento determinado libera el excedente energético emitiendo rayos beta y gama. El espectro de rayos gama generado es medido mediante un reactor semiconductor. La medición y cuantificación de los elementos químicos se realiza a partir de los picos generados en este espectro, en el cual el área es proporcional a la cantidad de ese elemento. Los datos generados necesitan ser correlacionados estadísticamente, lo que puede lograrse a través de un análisis de componentes principales y/o un análisis de cluster, ambos orientados a determinar la estructura de los datos (Bishop et al. 1982; Bishop y Neff 1989).
} 
Tabla 1. Características del conjunto cerámico analizado por AAN $(n=50)$. Modificado de Páez y Plá (2015). Referencias: SM N/ BI: Santa María negro sobre blanco; SM N y R / BI: Santa María negro y rojo sobre blanco; SM N / R: Santa María negro sobre rojo; Famabalasto NG: Famabalasto negro grabado; Famabalasto N / R: Famabalasto negro sobre rojo.

Table 1.Characteristics of the ceramic set analyzed by AAN $(n=50)$. Modified from Páez and Plá (2015). References: SM N I BI: Santa María black on white; SM N and R / Bl: Santa María black and red on white; SM N / R: Santa Maria black on red; Famabalasto NG: Engraved black Famabalasto; Famabalasto N / R: Black on red Famabalasto.

\begin{tabular}{|c|c|c|c|c|}
\hline \multirow[t]{2}{*}{ Sitio } & \multirow[t]{2}{*}{$\mathbf{N}$} & \multicolumn{2}{|c|}{ Estilo cerámico } & \multirow[t]{2}{*}{ Forma de la pieza } \\
\hline & & $n$ & Clasificación & \\
\hline \multirow{3}{*}{ S.Tuc.Tav. 15} & \multirow{3}{*}{20} & 15 & $\mathrm{SM} N / \mathrm{BI}$ & Cerrada, abierta, indet. \\
\hline & & 2 & SM N y R / BI & Cerrada \\
\hline & & 3 & Tosco & Cerrada \\
\hline \multirow{8}{*}{ LCZVIIIS1 } & \multirow{8}{*}{30} & 5 & $\mathrm{SM} \mathrm{N} / \mathrm{BI}$ & Cerrada, abierta \\
\hline & & 1 & SM N y R / BI & Cerrada \\
\hline & & 4 & Tosco & Cerrada \\
\hline & & 1 & SM N / R & Cerrada \\
\hline & & 2 & Famabalasto NG & Abierta \\
\hline & & 5 & Famablasto N / R & Abierta \\
\hline & & 2 & Yocavil & Abierta \\
\hline & & 10 & Inca & Cerrada, abierta \\
\hline
\end{tabular}

plasticidad. Por encima del estrato hay niveles de loess y presencia de iluviación. La muestra extraída $(\mathrm{M} 2)$ es de color 10R 5/5, textura arcillosa y muy plástica.

Sector 3 (S3): Se encuentra a unos pocos kilómetros de distancia del S2, frente al actual dique, sobre el faldeo de la Loma de La Angostura, junto a la ruta provincial Nº 307. El depósito está asociado a una zona de movilización del basamento metamórfico. La muestra obtenida (M3) es de color 10R 5/6 y textura arcillosa (Figura 2).

Sector 4 (S4): Identificamos este depósito en la ladera NE del cerro Ñuñorco, en un lugar llamado
Mula Corral al que llegamos a través de una senda de animal (senda La Hoyada). Se trata de una quebrada de pendiente pronunciada (80\% - 90\%) donde afloran rocas metamórficas de grano fino. En las proximidades transcurre el río Mula Corral. El depósito arcilloso tiene 5 $\mathrm{m}$ de alto y $8 \mathrm{~m}$ de ancho, con clastos rodados visibles a simple vista. La muestra extraída (M4) tiene una textura arcillosa de color 10R 4/4.

Sector 5 (S5): Se encuentra en el valle de Las Carreras, al pié de la cañada del río Muñoz, hacia la margen derecha. En la superficie se identificó un manchón muy leve, delimitado por vegetación. Se cotejó en el perfil de una

Tabla 2. Ubicación y características de los sectores de muestreo y muestras obtenidas, vinculación con las actividades económicas actuales y evidencias arqueológicas registradas en las proximidades.

Table 2. Location and characteristics of the sampling sectors and samples obtained, link with current economic activities and archaeological evidence registered in the vicinity.

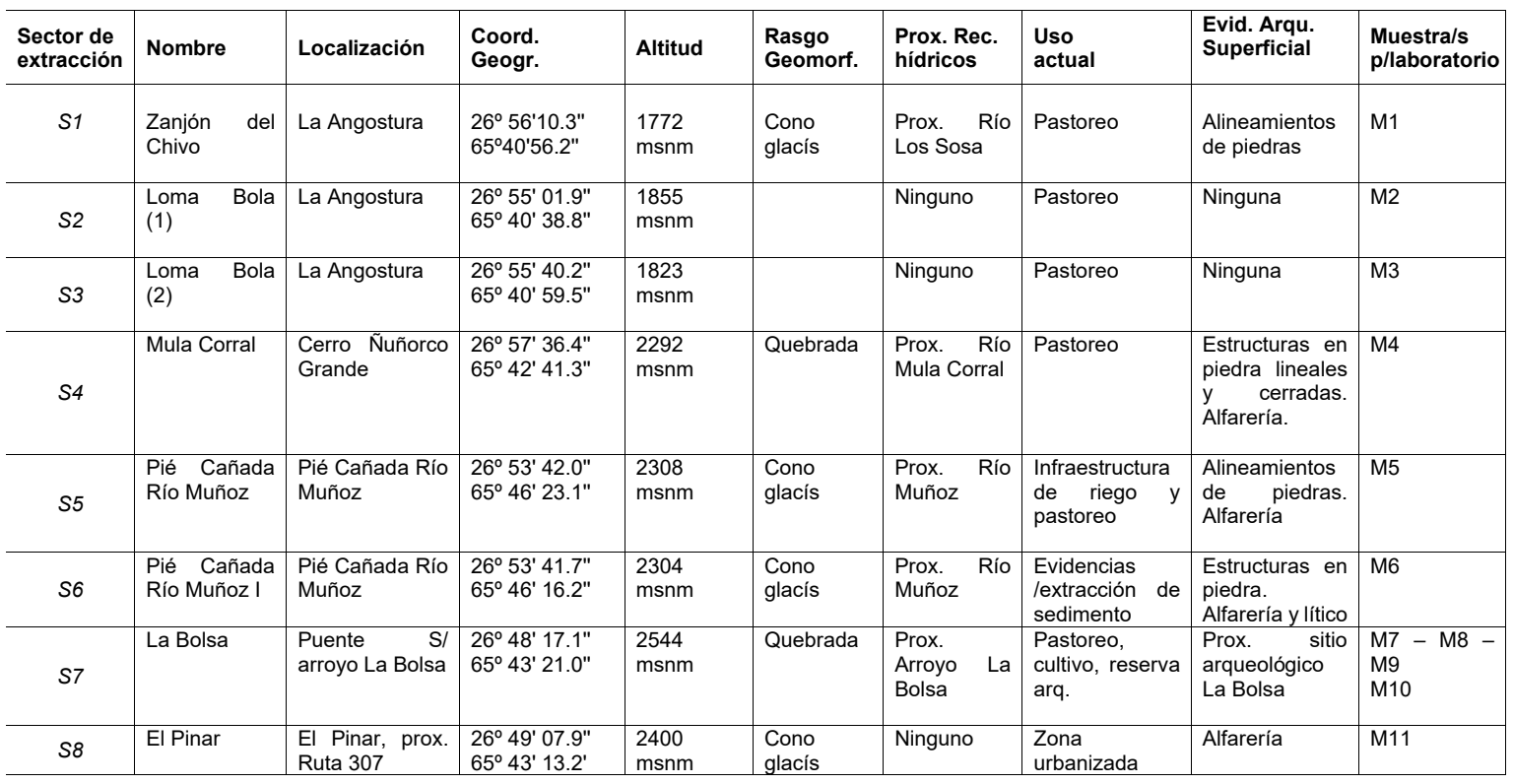



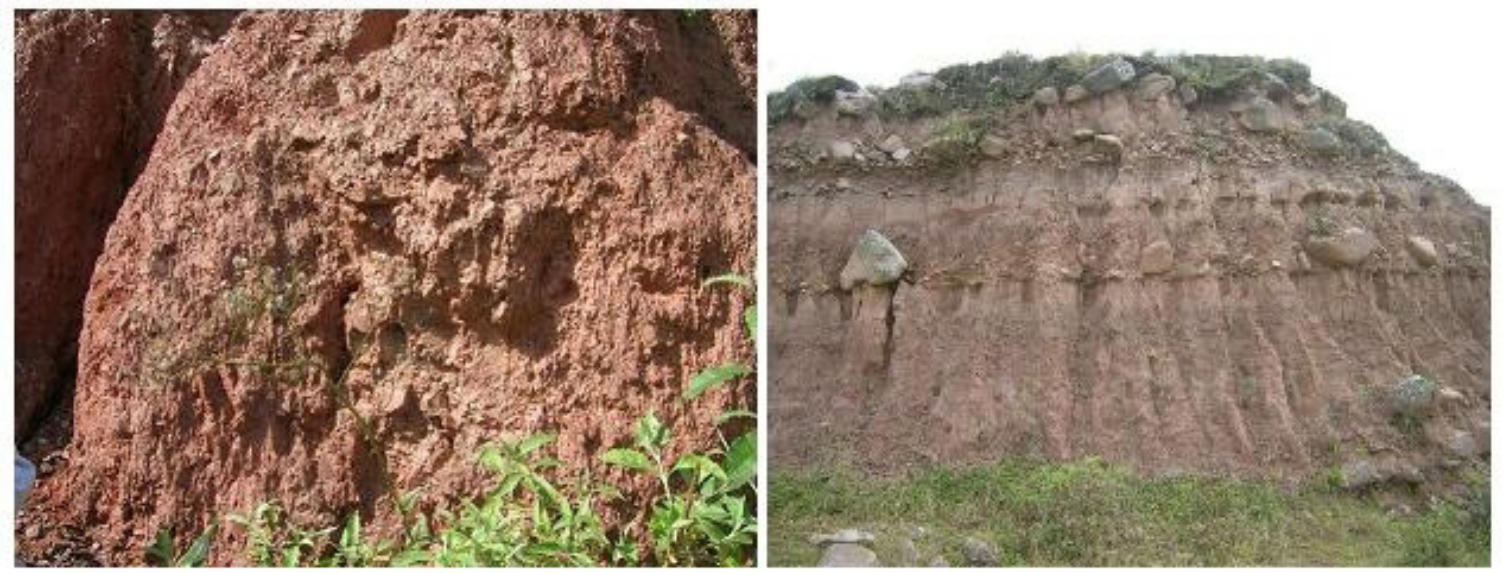

Figura 2. Derecha: Depósito arcilloso asociado a la zona de movilización del basamento metamórfico de donde se obtuvo la M3. Izquierda: Paleosuelo del perfil La Bolsa, del cual proceden las muestras M7, M8, M9 y M10.

Figure 2. Right: Clay deposit associated with the mobilization zone of the metamorphic basement from which the M3 was obtained. Left: Paleo-soil of the La Bolsa profile, from which samples M7, M8, M9 and M10 come.

barranca próxima que correspondería al horizonte $\mathrm{B}$ del suelo que tiene de 0,15 a 0,20 m, donde hay evidencias de iluviación y macroporos conteniendo material suspendido (posiblemente BC y C). La excavación de unos centímetros subsuperficiales en el sector de extracción proporcionó un sedimento loéssico, con plasticidad y estructura limosa (M5).

Sector 6 (S6): A unos $300 \mathrm{~m}$ al $\mathrm{N}$ del S5 se identificó un sector superficial con evidencias de extracción de suelo que las alfareras del lugar indican como una fuente utilizada. El perfil edáfico obtenido en el campo describe un horizonte $A$ de estructura granular $(0,20 \mathrm{~m})$, seguido por un $A B(0,05-0,10 \mathrm{~m})$, un $B$ con acumulación iluvial de materia orgánica y arcilla, Bht $(0,20 \mathrm{~m})$ y finalmente un horizonte $\mathrm{Bt}$, es decir, argílico. De este último se obtuvo la muestra 6 (M6), de textura arcillosa.

Sector 7 (S7): Este sector se encuentra al costado del puente sobre el arroyo La Bolsa, en una quebrada en el piedemonte de las Cumbres Calchaquíes, próximo al sitio arqueológico que lleva el mismo nombre. En este perfil, trabajado anteriormente por Collantes (2001), se obtuvieron tres muestras sedimentológicas correspondientes a distintos niveles de paleosuelo. La muestra 7 (M7) es un sedimento arcilloso muy plástico, de color 5YR 4/4, asignado al paleosuelo inferior del miembro superior descripto por Collantes, cuyo espesor alcanza los 0,30-0,40 m. La muestra 8 (M8) tiene una textura limo-arenosa, de color algo más intenso que 10YR 5/4. Correspondería a un paleosuelo localizado por encima de donde se extrajo la Muestra 7. Este estrato tiene un espesor aproximado de 0,40 m. La muestra 9 (M9) es un sedimento limo-arenoso, de color 10YR 5/4, localizado por debajo de la Muestra 7. El paleosuelo que lo contiene tiene una potencia de 0,25 m. A $120 \mathrm{~m}$ arroyo adentro sobre el perfil La Bolsa y 1,20 m. del nivel de la superficie se extrajo otra muestra sedimentaria (M10), que parece corresponder al mismo sedimento de la M7, pero con mayor cantidad de minerales ferruginosos. El análisis posterior de laboratorio nos aclarará las relaciones entre ambas muestras (Figura 2).

Sector 8 (S8): Hacia el norte de la Villa de Tafí, también en las Cumbres Calchaquíes, en el área conocida como El Pinar, se encuentra el último sector muestreado, al que llegamos por referencia de un poblador local. Se trata de la parte final del cono glacís atravesado por la ruta 307, en cuyo costado identificamos un sedimento de coloración oscura 5YR 3/3 y textura arcillosa que constituyó la muestra sedimentaria 11 (M11).

Los análisis de Difracción de Rayos $\mathrm{X}$, realizados sobre las muestras procedentes de los sectores mencionados, indicaron una mayor proporción de caolinita y en proporciones menores, illita, registrando en una única muestra la presencia de esmectita (Tabla 3). Para el caso de las illitas, hay una diferencia muy marcada en sus índices de plasticidad (IP) que, en el caso de la muestra del Río Muñoz (nivel de suelo activo) alcanza un valor de 13.92, en tanto en La Bolsa (paleosuelo) sólo llega a 3.83. En ambos, por tratarse de niveles edafizados, se trata de illitas degradadas, que han experimentado pérdida parcial de $\mathrm{K}$ y por ello han adquirido mayor plasticidad. Esto refleja la falta de correlación entre el comportamiento geomecánico y la mineralogía de las arcillas. Es importante destacar la presencia de vidrio volcánico en la muestra de El Pinar (M11), en consonancia con los datos regionales proporcionados por Cremonte (1996), quien refiere su presencia tanto en las arcillas como en las arenas procedentes del altivalle de La Ciénaga.

Los valores obtenidos de IP y COLE para las 11 muestras analizadas sugieren que sólo en un caso -muestra 1 Zanjón del Chivo-, las características del sedimento no permiten su 

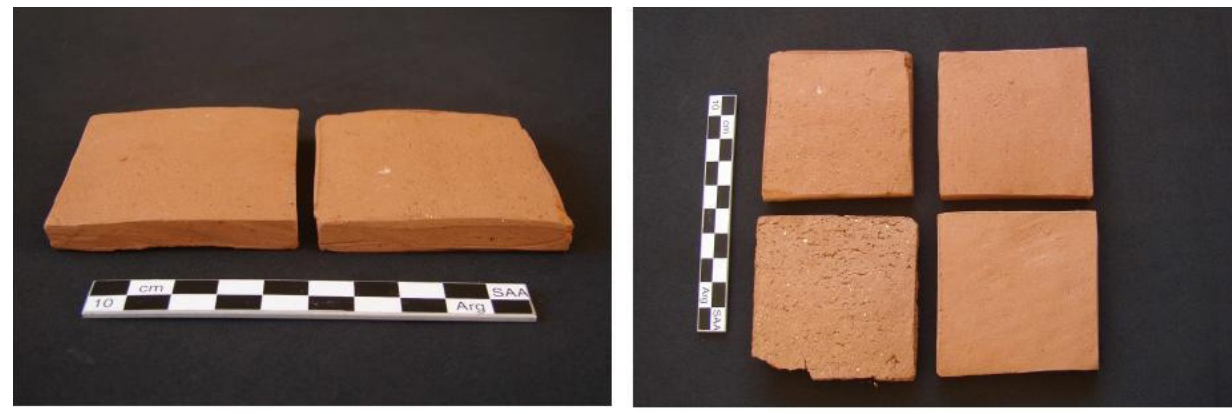

depósitos que presentan condiciones geomecánicas favorables para la producción alfarera, según se detalló en el acápite anterior, elaborándose briquetas experimentales.

Se realizó, así, el análisis y la comparación del perfil químico de los fragmentos

Figura 3. Briquetas experimentales con 4 de las arcillas muestreadas y analizadas.

Figure 3. Experimental briquettes with 4 of the clays sampled and analyzed.

empleo en la manufactura cerámica (IP=3.73; COLE=3.07) [Tabla 3]. El resto de los casos mostraron valores positivos, variables dentro del conjunto. Las muestras procedentes de Loma Bola, Mula Corral y el río Muñoz son las que manifestaron mayor grado de plasticidad. Esto permite obtener un cuadro distribucional de arcillas que cubre los distintos sectores del Valle, algunos de ellos muy próximos a los contextos arqueológicos mencionados anteriormente.

\section{Correlación química entre arcillas y fragmentos cerámicos prehispánicos}

A partir de los resultados de nuestros estudios se seleccionaron 4 muestras $(n=4)$ correspondientes a los seleccionados de los sitios S.Tuc.Tav. 15 y LCZVIIIS1 ( $n=50)$ y de las briquetas elaboradas con las arcillas $(n=4)$ de los depósitos de la Loma Bola (M3), La Bolsa (M7 y M10) y El Pinar (M11) a través de análisis de Activación Neutrónica (AAN).

El Análisis de Componentes Principales (ACP) permitió obtener cinco componentes que explican el 76,60\% de la variación total. Sobre esta base se efectuó un análisis de conglomerados jerárquicos, utilizando el método de vinculación promedio Ward y la distancia euclideana al cuadrado para identificar grupos con perfil químico similar, que fueron ajustados utilizando la distancia Mahalanobis (Ratto et al., 2007).

Inicialmente se identificaron tres grupos predictivos (GP), que luego fueron reducidos a dos, GP1 y GP2 (Figura 4). Ambos grupos combinan fragmentos de los dos sitios prehispánicos, pero sólo el GP1 muestra relación con las fuentes de arcilla. Este concentra gran parte de los fragmentos Famabalasto negro sobre rojo

Tabla 3. Resultados del análisis geomecánico y mineralógico de las muestras de arcilla recolectadas. Fco.= Franco, Arc. $=$ Arcilloso, Are.= Arenoso, $\mathrm{II}=\mathrm{Illita}, \mathrm{Caol}=$ Caolinita, Expand $=$ Expandibles, $\mathrm{S}=$ Esmectita

Table 3. Results of the geomechanical and mineralogical analysis of the collected clay samples. Fco. = Loam, Arc. =Clay, Are. $=$ Sandy, $I I I=I I$ ite, $\mathrm{Caol}=$ Kaolinite, Expand $=$ Expandable, $S=$ Smectite

\begin{tabular}{|c|c|c|c|c|c|c|c|c|}
\hline Muestra & Ubicación & $\begin{array}{c}\text { Clase } \\
\text { textural }\end{array}$ & LP & LL & IP & COLE & Tipo de arcilla & Material \\
\hline M1 & Zanja del Chivo & Fco. & 23.01 & 26.74 & 3.73 & 3.07 & Illita & Cuaternario \\
\hline M2 & La Angostura & $\begin{array}{ll}\text { Fco. } & \text { Arc. } \\
\text { Are. } & \end{array}$ & 24.69 & 29.60 & 4.91 & 3.84 & Caol. - III. Abierta+Expandibles & Regolito \\
\hline M3 & Loma Bola & Arc. & 25.70 & 52.65 & 26.94 & 5.88 & $\begin{array}{l}\text { III abierta+ } \\
\text { Expandibles+ } \\
\text { Caol (vestigios) }\end{array}$ & Regolito \\
\hline M4 & Mula Corral & Arc. Are. & 18.76 & 34.30 & 15.63 & 4.28 & $\begin{array}{ll}\text { III+Expand } & \text { (vestigios)+Caol } \\
\text { (vestigios) } & \end{array}$ & $\begin{array}{l}\text { Asociado a metamorfitas - relictos } \\
\text { arcillosos }\end{array}$ \\
\hline M5 & Río Muñoz & Fco. Arc. & 19.73 & 33.65 & 13.92 & 7.69 & $\begin{array}{lll}\begin{array}{l}\text { III+Expand } \\
\text { (vestigios) }\end{array} & \text { (vestigios)+ } & \text { Caol } \\
& & \end{array}$ & Suelo actual, horizonte B \\
\hline M6 & Río Muñoz & Fco. Arc. & 20.31 & 36.91 & 16.60 & 9.09 & $\begin{array}{lll}\text { III+Expand } & \text { (vestigios)+ } & \text { Caol } \\
\text { (vestigios) } & & \end{array}$ & Suelo actual, horizonte B \\
\hline M7 & La Bolsa & Fco. Arc. & 20.49 & 28.63 & 8.13 & 4.76 & III+Expand (vestigios) & $\begin{array}{l}\text { Sedim. arcilloso muy plástico - } \\
\text { paleosuelo }\end{array}$ \\
\hline M8 & La Bolsa & $\begin{array}{ll}\text { Fco. } & \text { Arc. } \\
\text { Are. } & \end{array}$ & 20.10 & 23.94 & 3.83 & 4.93 & III-S+Expandibles (vestigios) & Sedim. limo arenoso - paleosuelo \\
\hline M9 & La Bolsa & Fco. Arc. & 19.24 & 28.00 & 8.76 & 6.66 & III+Expandibles (vestigios) & Sedim. limo arenoso - paleosuelo \\
\hline M10 & La Bolsa & Arc. & 20.31 & 32.03 & 11.72 & 7.69 & III+Expandibles & $\begin{array}{l}\text { Sedim. arcilloso muy plástico - } \\
\text { paleosuelo }\end{array}$ \\
\hline M11 & El Pinar & Arc. & 21.64 & 31.22 & 9.57 & 5.47 & III+Expandibles+Vidrio & Cuaternario \\
\hline
\end{tabular}


y Yocavil, además de un número no menor de pastas Santa María negro sobre blanco del contexto tardío y algunos fragmentos toscos ${ }^{6}$, no pintados. En el GP2 están asociados la mayor parte de los fragmentos Santa María de ambos contextos junto a las Famabalasto negro grabado y casi todos los fragmentos con decoración inca. Por su parte, las variantes tricolor y bicolor negro sobre rojo del estilo Santa María se asocian al GP2, que es el que concentra la mayor parte de los materiales del contexto LCZVIIIS1. Por fuera de ambas elipses se ubican 4 fragmentos, 2 correspondientes al estilo Santa María del contexto tardío, y dos al estilo inca del contexto homónimo (Tabla 4, Figura 3) [Páez y Plá 2015].

Hay que destacar dos aspectos de particular interés, que retomaremos posteriormente: 1) la relación de las arcillas con los fragmentos Famabalasto negro sobre rojo y Yocavil, 2) la asociación química de la mayor parte de los materiales Santa María de ambos contextos con los fragmentos de estilo inca, pero con ninguna de las fuentes de arcilla analizadas.

Un dato para considerar es la cercanía espacial de estas últimas con los sitios arqueológicos considerados. Para el caso de S.Tuc.Tav.15, la distancia a la fuente de la cual se extrajo la muestra M3 es de $15 \mathrm{~km}$, en tanto otras zonas muestreadas se encuentran más próximas (de 3 a $10 \mathrm{~km}$ ). Para el caso de LCZVIIIS1, las muestras M7 y M10 se ubican a 2,5 km, en tanto M11 está a 3,5 km. Para el caso de la muestra M3, la distancia se amplía a casi $10 \mathrm{~km}$.

\section{Discusión de los resultados y reflexiones finales}

En primer lugar, es de destacar la correlación positiva entre las cuatro fuentes de arcilla y los fragmentos correspondientes a ambos contextos arqueológicos en igual proporción numérica, es decir, más allá de las distancias a las mismas.

En segundo lugar, atendiendo a los resultados acerca del Grupo Predictivo 1 (GP1) - que concentra una importante diversidad estilístico-tecnológica asociada a las arcillas locales -, se puede inferir el uso de las mismas fuentes de materia prima para elaborar piezas que fueron trabajadas con diferentes técnicas y que probablemente, hayan tenido diferentes funciones. Ello es aún más notorio en el caso de las vasijas sin pintura denominadas toscas?

\footnotetext{
${ }^{6}$ Referimos como "toscos", para sintetizar, aquellos fragmentos de piezas que no presentan un tratamiento superficial con pintura o agregado de apliques. Suelen presentar superficies alisadas o cepilladas. ${ }^{7}$ De acuerdo a los análisis submacroscópicos y microscópicos realizados sobre ese tipo de fragmentos del contexto S.Tuc.Tav.15, se trata de vasijas elaboradas a partir de una mezcla de arcillas y arenas, asegurando objetos resistentes a la fractura (Páez, 2010). Las características del contexto de hallazgo hacen suponer que podrían haber funcionado como contenedores de restos humanos, o que habrían formado parte del ajuar asociado al evento funerario. Por otra parte, las mismas fuentes se correlacionan con piezas pintadas, de menor espesor, que fueron elaboradas sin adición de antiplástico, eliminando del cuerpo arcilloso los clastos de tamaño medio-grueso. Los fragmentos Famabalasto
}

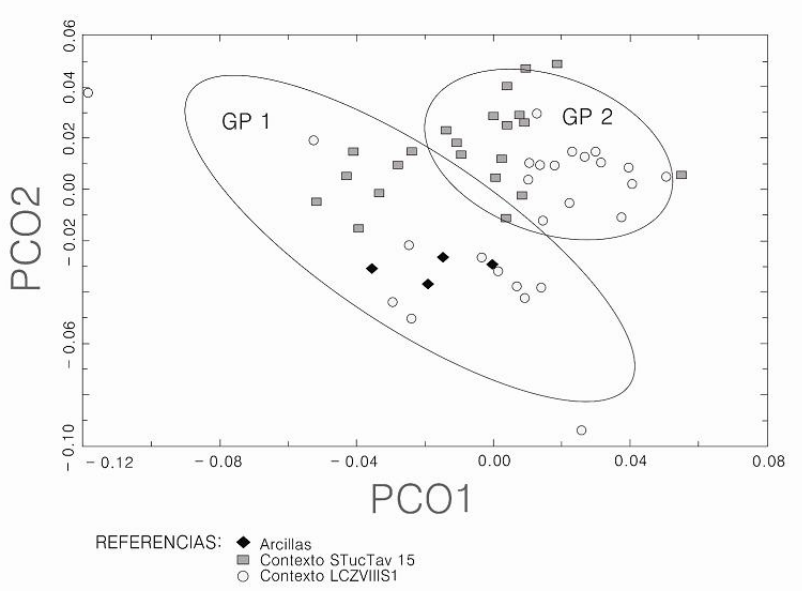

Figura 4. Agrupamiento de arcillas y fragmentos en el espacio factorial definido por los factores 1 y 2 . $n=54$. Tomado de Páez y Plá (2015).

Figure 4. Grouping of clay samples and sherds in the factorial space defined by factors 1 and 2. $n=54$. Taken from Páez and Plá (2015).

Entonces, al menos tentativamente, podemos inferir que las sociedades que están habitando el Valle de Tafí hacia mediados del segundo milenio de la EC utilizaron fuentes locales de aprovisionamiento para la elaboración de piezas diferentes entre sí, tanto en lo que hace a las características de su manufactura como a los contextos en los que parecen haber funcionado.

En ese marco, es interesante la correlación positiva (GP1) entre las arcillas de las fuentes locales y casi la totalidad de los fragmentos Famabalasto negro sobre rojo y Yocavil polícromo. Tomando en cuenta las discusiones que evalúan tanto la procedencia como los procesos sociales y políticos implicados en estos estilos alfareros, estaríamos frente a la posibilidad de una producción local de al menos algunas piezas cerámicas, más allá de las particularidades de sus procesos de manufactura (Cf. Páez 2010). Estas afectan principalmente al tratamiento de las arcillas para la preparación del cuerpo plástico (menor tamaño de granos, muy buena selección de los minerales), y la ausencia de carga piroclástica que se pudo identificar en un porcentaje importante de materiales cerámicos de LCZVIIIS1 (Páez y Arnosio, 2009).

Así, tomando en cuenta la correlación química de los fragmentos con las arcillas locales, podemos inferir que las escudillas Famabalasto N/R y Yocavil pueden haber sido elaboradas en el valle de Tafí, más allá de que presentan diferencias ciertamente significativas en su perfil técnico de manufactura y modalidad estilística con la alfarería del Tardío local, de estilo predominantemente santamariano. No estamos en condiciones de avanzar en hipótesis acerca

N/R y Yocavil corresponden a escudillas que habrían formado parte de espacios rituales/festivos de LC ZVIIIS1, que nada tienen que ver con el contexto mortuorio con el que se asocian los materiales toscos referidos 
Tabla 4. Conformación de los grupos predictivos (GP), sobre un $n=50$. Restan 4 fragmentos que no se asocian a ningún GP. Adaptado de Páez y Plá (2015).

Table 4. Conformation of predictive groups (GP), on $n=50$. There are 4 fragments that are not associated with any GP. Adapted from Páez and Plá (2015).

\begin{tabular}{|c|c|c|c|c|}
\hline \multirow{2}{*}{$\begin{array}{l}\text { Grupo } \\
\text { Predictivo }\end{array}$} & \multirow[t]{2}{*}{ Arcillas } & \multirow[t]{2}{*}{ Contexto } & \multicolumn{2}{|c|}{ Fragmentos } \\
\hline & & & $\mathrm{n}$ & Estilo decorativo \\
\hline \multirow[t]{6}{*}{1} & \multirow[t]{6}{*}{4} & \multirow[t]{2}{*}{ STuc.Tav.15 } & 5 & Santa María N/BI \\
\hline & & & 2 & Toscos \\
\hline & & \multirow[t]{4}{*}{ LCZVIIIS1 } & 4 & Famabalasto N/R \\
\hline & & & 2 & Yocavil \\
\hline & & & 1 & Inca \\
\hline & & & 2 & Toscos \\
\hline Total & 4 & & 16 & \\
\hline \multirow[t]{9}{*}{2} & \multirow[t]{9}{*}{0} & \multirow[t]{3}{*}{ STuc.Tav.15 } & 9 & Santa María N/BI \\
\hline & & & 1 & $\begin{array}{l}\text { Santa María N y } \\
\mathrm{R} / \mathrm{BI}\end{array}$ \\
\hline & & & 1 & Toscos \\
\hline & & \multirow[t]{6}{*}{ LCZVIIIS1 } & 5 & Santa María N/BI \\
\hline & & & 1 & Santa María N/R \\
\hline & & & 1 & $\begin{array}{l}\text { Santa María N y } \\
\text { R/BI }\end{array}$ \\
\hline & & & 2 & Famabalasto NG \\
\hline & & & 1 & Famabalasto N/R \\
\hline & & & 9 & Inca \\
\hline Total & 0 & & 30 & \\
\hline
\end{tabular}

de quienes produjeron estas piezas: población nativa local (siguiendo ciertos prototipos de producción alfarera) o población trasladada - trabajadores especializados - en el marco del dominio inca de la región (Lorandi, 1980; Taboada et al., 2013; Williams, 1983). Los resultados de nuestros estudios refuerzan algo que ya se está conociendo para otros asentamientos contemporáneos (Williams y Cremonte 1994): la producción de este tipo de piezas con materias primas locales. Es necesario contemplar, sin embargo, que a diferencia de sitios como Ingenio del Arenas Médanos o Potrero Chaquiago, el caso que presentamos en este trabajo es el de un asentamiento con fuerte relevancia de ocupación local.

Por otro lado, hay un número importante de fragmentos que se conglomera en el GP2, sin relación con las cuatro fuentes de arcilla incorporadas al análisis. Estos materiales corresponden a los dos contextos: $n=11$ a S.Tuc.Tav.15 y $n=19$ a LCZVIIIS1. En este grupo se incluyen la mayor parte de los fragmentos Santa María de ambos sitios y casi la totalidad de los incaicos. Cabe preguntarnos, entonces, ¿cuáles serían los depósitos utilizados para la manufactura de estas piezas?, si bien podemos concluir que a pesar de no ser contemporáneos, las fuentes utilizadas podrían estar relacionadas. En principio, se podrían esperar resultados positivos al incorporar alguna/s de las otras fuentes muestreadas que dieron cuenta de su aptitud para el uso alfarero (Tabla 3), pero también podría ocurrir que el aprovisionamiento se realice a partir de depósitos más allá de los límites del valle, no considerados en este trabajo. Al respecto es importante considerar las similitudes identificadas por Cremonte et al. (1991) entre las arcillas de La Ciénaga y Tafí del Valle, lo que habría determinado importantes vinculaciones en la producción alfarera de ambos espacios, en este caso durante el primer milenio EC.

Finalmente, y para concluir, es importante destacar que, amén de que la muestra es limitada numéricamente, consideramos que los resultados obtenidos son concluyentes. La observación de estos datos a la luz de una muestra mayor de fragmentos, así como la incorporación de otras fuentes de arcilla a los análisis, representa un desafío a futuro. Ello permitiría despejar interrogantes generados, por ejemplo, en torno al conjunto de materiales cerámicos de ambos sitios que no pudo afiliarse composicionalmente a ninguno de los depósitos plásticos analizados.

La Plata, 28 de agosto de 2019

\section{Agradecimientos}

Diversas personas e instituciones hicieron posible este trabajo. La Dra. Patricia Cuenya y la Geol. Ramona Ovejero de Indri colaboraron en los trabajos de campo y laboratorio, la comunidad de Tafí del Valle y las autoridades municipales vienen acompañando los trabajos de investigación en el Valle y diversos alumnos y egresados participaron en las tareas de excavación a partir de las cuales se recuperaron los fragmentos cerámicos que aquí analizamos. La Universidad Nacional de Catamarca, La Universidad Nacional de Tucumán y el Consejo Nacional de Investigación Científicas y Técnicas representaron un apoyo académico y financiero fundamental en las distintas instancias de la investigación. Amén a todo ello, la responsabilidad por lo expresado es exclusiva de las autoras.

\section{Bibliografía}

Bishop, Ronald L., Rands, R. L. y Holley, G. (1982) Ceramic Compositional Analysis in Archaeological Perspective. En: Advances in Archaeological Method and Theory 5. Schiffer, M. (Ed.). New York: Academic Press, pp. 275-330.

Bishop, R. L. y H. Neff (1989) Compositional data analysis in archaeology. En: Archaeological Chemistry IV. Advances in Chemistry Series 220. Allen, R. O. (Ed.). Washington D.C: The American Chemical Society, pp. 57-86.

Brasher B., Franzmeier D., Valassis V. y Davidson, S. (1966) Use of Saran resin to coat natural soil clods for Bulk-density water retention measurements. Soil Science Vol. 101, p. 108. 
Calderari, M. y Williams, V. (1991) Re-evaluación de los estilos cerámicos incaicos en el noroeste argentino. Comechingonia 9, Nro. Especial, $73-95$.

Collantes, M. (2001) Paleomorfología y geología del Cuaternario de la Cuenca del Río Tafí, Departamento de Tafí del Valle, Provincia de Tucumán, Argentina. Tesis Doctoral inédita. Universidad Nacional de Salta.

Collantes, M., Powell, J.E. y Sayago, J.M. (1993) Formación Tafí del Valle (Cuaternario superior), provincia de Tucumán (Argentina): litología, paleontología y paleoambientes. Actas del $12^{\circ}$ Congreso Geológico Argentino y $2^{\circ}$ Congreso de Exploración de Hidrocarburos (Buenos Aires), Tomo 2, 200206.

Cremonte, M. B. (1996) Investigaciones arqueológicas en la Quebrada de la Ciénaga. (Dpto. de Tafí, Tucumán). Tesis Doctoral inédita. Facultad de Ciencias Naturales y Museo, Universidad Nacional de La Plata.

Cremonte, M. B., Flegenheimer, N., Plá, R., Cohen, M. y Gordon, A. (1991) Aplicación del método de Activación de Neutrones (NAA) en cerámicas arqueológicas del Noroeste de Argentina. Revista del Instituto de Geología y Minería 8: 53-70.

Cremonte, M. B. y Pereyra Domingorena, L. (2013) Atlas de pastas cerámicas arqueológicas. Petrografía de estilos alfareros del NOA. Jujuy: Editorial de la Universidad Nacional de Jujuy.

Cuenya, P., Ovejero, R., Manasse, B. y Páez, M. C. (2007) Materias primas para cerámica ¿probables fuentes? Ponencia presentada en VIII Jornadas de Comunicaciones de la Facultad de Ciencias Naturales e Instituto Miguel Lillo, Universidad Nacional de Tucumán, Tucumán.

De Pablo, L. (1964) Las Arcillas. Clasificación, Identificación, Usos y Especificaciones Industriales. Boletín de la sociedad Geológica Mexicana T. XXVII, 2, 49-92.

Fernández, R. (1995) Informe Geoindustrial de los depósitos cineríticos del sector industrial del Valle de Tafí, Provincia de Tucumán. Tucumán: UNSTA. M. S.

lucci, E. (2013) Producción, circulación y uso de cerámica tardía en el Valle de Hualfín (Catamarca, Argentina). Tesis doctoral inédita. Facultad de Ciencias Naturales y Museo, Universidad Nacional de La Plata.

Krauss, E., Hunt ,W., y Ramsdell, L. (1965) Mineralogía. Madrid: Ed. Castilla S.A.

Leiva Benegas, J. (2003) Analysis of Possible Pre-Selection Patterns Regarding to Cementeries in Calchaqui Valley, Tucumán, Argentine. Tesis de Licenciatura inédita, Göteborgs Universitet Institutionen för arkeologi C-uppsats, Göteborg.

Lorandi, A. M. (1980) La frontera oriental del Tawantinsuyu: el Umasuyu y el Tucumán. Una hipótesis de Trabajo. Relaciones de la Sociedad Argentina de Antropología XIV (1), 147-164.

Manasse, B. (2003) Arqueología en los faldeos suroccidentales de las Cumbres Calchaquíes. Aportes Científicos desde Humanidades 3, 393-409.

Manasse, B. (2012) Arqueología en el borde andino del Noroeste Argentino: sociedades del último milenio en el Valle de Tafí. Tesis doctoral inédita. Facultad de Ciencias Naturales y Museo, Universidad Nacional de La Plata.

Manasse, B. (2014a) Hacia el oriente de la región valliserrana: historia diaguita de Tafí del Valle, Tucumán, Revista Arqueología 20 Dossier, 217-239.

Manasse, B. (2014b) El pasado indígena tafinisto: Analizando los tiempos del imperio inca. Actas de las $1^{a}$ Jornadas Regionales y $3^{a}$ Internas de Antropología del NOA. Universidad Nacional de Salta, pp. 378 - 396.

Manasse, B. (2017) Diaguitas, Tafíes, Tafinistos y Tafinistas: un mundo abigarrado de identidades, historias y territorios. En: Historia del Municipio de Tafí del Valle. Noli, E. (Coord.). Buenos Aires: Ediciones Imago Mundi, pp. 1-63.

Manasse, B. (2019) Arqueología en el Valle de Tafí (provincia de Tucumán): algunas miradas sobre el pasado-presente de su gente, Revista del Museo de La Plata 4(1), pp. 121-143. https://doi.org/10.24215/25456377e072

Marchegiani, M., Palamarczuk, V. y Reynoso, A. (2009) Las urnas negro sobre rojo tardías de Yocavil (Noroeste argentino): Reflexiones en torno al estilo. Bol. Mus. Chil. Arte Precolomb. 14 (1): 69-98.

Núñez Regueiro, V. (1972) Conceptos teóricos que han obstaculizado el desarrollo de la arqueología en Sudamérica. Estudios de Arqueología No 1: 11-35.

Páez, M. C. y Giovannetti, M. (2008). Intersecciones y Síntesis. Sincretismos en los platos del Período Inkaico del Noroeste Argentino. Revista Arqueología Sudamericana. Vol. 4 (2): 169-190.

Páez, M. C. y Giovannetti, M. (2009) Tipologizando identidades. Reflexiones sobre la construcción de identidades étnicas en la Arqueología del NOA. Revista Avá No 13: 155-170. Facultad de Humanidades y Ciencias Sociales, Universidad Nacional de Misiones. Posadas.

Páez, M. C. y Arnosio, M. (2009) Inclusiones piroclásticas en pastas cerámicas del valle de Tafí (Tucumán, Argentina): implicancias para las prácticas de producción. Estudios Atacameños $\mathrm{N}^{\circ}$ 38, 5-20.

Palamarczuk, V., Reynoso, V. y Marchegiani, M. (2014) Estudio sobre las pastas de las urnas Negro sobre Rojo tardías de Yocavil, Noroeste argentino. Una primera aproximación. Revista Comechingonia 18 (1), 161-168.

Patané Aráoz, C. J. (2008) Arqueología de los Encuentros: Lo Inka y lo Local en un sitio en las montañas de un valle del NOA. Estudiando al Pukara de las Lomas Verdes (Tafi del Valle, Prov. de Tucumán). Oxford: South American Archaeological Series No 9 Ed. Por Izeta, A. D. BAR International Series. 
Pérez, M. y Tchilinguirian, P. (2016) Petrografía cerámica de la alumbrera: aportes al problema de la tecnología y la procedencia (Antofagasta De La Sierra, Puna Austral Argentina), Cuadernos del Instituto Nacional de Antropología y Pensamiento Latinoamericano 25, $75-93$.

Prieto Olavarría, C. y Páez, M. C. (2015) Presencia de inclusiones piroclásticas en la cerámica de los siglos XV a XVII en el Centro Oeste y Noroeste Argentino. Chungara Revista de Antropología Chilena 47 (3), 441-453.

Puente, V. (2011) Prácticas de producción alfarera en el Valle del Bolsón: materias primas y modos de hacer ca. 900-1600 DC. Tesis doctoral inédita, Facultad de Filosofía y Letras, Universidad de Buenos Aires.

Raffino, R. (1991) Poblaciones Indigenas en Argentina. Urbanismo y proceso social precolombino. Buenos Aires: Editorial TEA.

Raffino, R. (2007) Poblaciones Indigenas en Argentina. Urbanismo y Proceso Social Precolombino. Buenos Aires: Editorial Emecé.

Ratto, N., Feely, A. y Plá, R. (2007) La Producción Alfarera en el bolsón de Fiambalá (Departamento Tinogasta, Catamarca) y su Alcance Extra-Regional. En Ratto, N. y Cremonte, B. (eds.), Cerámicas Arqueológicas: Perspectivas Arqueométricas para su Análisis e Interpretación. San Salvador de Jujuy: Editorial de la Universidad Nacional de Jujuy, pp. 123-145.

Ruiz Huidobro, O. (1972) Descripción Geológica de la Hoja 11 E Santa María. Buenos Aires: Ministerio de Industria y Minería. Servicio Nacional Minero Geológico.

Santillán de Andrés, S. (1951) Poblaciones indigenas en el Valle de Tafí. Tucumán: Geographia una et varia.

Sempé, M. C. (1999) La cultura Belén. Actas del XII Congreso Nacional de Arqueología Argentina Vol. II. Universidad Nacional de La Plata. Pp. 250- 258.
Taboada, C. Angiorama, C. Leiton, D. y López Campeny, S. (2013) En la llanura y en los valles... Relaciones entre las poblaciones de las tierras bajas santiagueñas y el Estado Inca. Intersecciones en Antropología 14, 137-156.

Tarragó, M. y Núñez Regueiro, V. (1972) Un diseño de investigación arqueológica sobre el Valle Calchaquí: Fase Exploratoria, Estudios de Arqueología 2, 62-85.

Thomas, P., Baker, J. and Zelazny, L. (2000) An expansive soil index for predicting shrink-swell potential. Soil Sci. Soc. Am. J. $64,268-274$.

Turner, J.C. (1960) Estratigrafía de la Sierra de Santa Victoria y adyacencias, Boletín de la Academia Nacional de Ciencias Córdoba 41, 163-196.

Turner, J.C. (1972) Cordillera Oriental. En Leanza, A. F. (Ed.) Geología Regional Argentina. Córdoba: Publicación Especial Academia Nacional de Ciencias, pp. 117-142.

Vera, S., De La Fuente, G. y Rasmussen, K. (2019) Prácticas alfareras, tecnología y cronología durante los períodos Tardío e Inca en el sector meridional del Valle de Abaucán. Tradiciones y rupturas: el caso de Costa de Reyes $\mathrm{N}^{\circ} 5$ (Tinogasta, Catamarca, Argentina), Latin American Antiquity 30(1), 70-90.

Williams, V. (1983) Evidencia de actividad textil en el establecimiento incaico de Potrero Chaquiago. Relaciones de la Sociedad Argentina de Antropología xv, 49-59.

Williams, V. y Cremonte, M. B. (1994). “¿Mitmaqkuna o circulación de bienes? Indicadores de la producción cerámica como identificadores étnicos. Un caso de estudio en el NOA". Avances en Arqueología 2: 9-27.

Zagorodny, N., M. Morosi, M. E. lucci y Wynveldt, F. (2010) Estudios composicionales de las pastas de cerámica tardía del Valle de Hualfín (Belén. Catamarca). Arqueología 16: 125-150. 